\title{
Evaluating multiloop Feynman integrals by Mellin-Barnes representation
}

\author{
V.A. Smirnov ${ }^{a *}$ \\ a Nuclear Physics Institute of Moscow State University, \\ Moscow 119992, Russia
}

The status of analytical evaluation of double and triple box diagrams is characterized. The method of MellinBarnes representation as a tool to evaluate master integrals in these problems is advocated. New MB representations for massive on-shell double boxes with general powers of propagators are presented.

\section{Introduction}

When calculating physical quantities that describe a given process one needs to evaluate a lot of Feynman integrals. After a tensor reduction based on some projectors (see, e.g., 1) a given Feynman graph generates various scalar Feynman integrals that have the same structure of the integrand with various distributions of powers of propagators. A straightforward analytical strategy is to evaluate, by some methods, every scalar Feynman integral generated by the given graph. If the number of these integrals is small such strategy is reasonable. In non-trivial situations, where the number of different integrals can be at the level of hundreds and thousands, it is reasonable to follow a well-known advanced strategy: to derive, without calculation, and then apply integration by parts (IBP) 2] and Lorentz-invariance (LI) 3] identities between the given family of Feynman integrals as recurrence relations. The goal of this procedure is to express a general integral from the given family as a linear combination of some basic (master) integrals. Therefore the whole problem of evaluation is decomposed into two parts: solution of the reduction procedure and evaluation of the master Feynman integrals.

There were several recent attempts to make the reduction procedure systematic:

(i) Using the fact that the total number of IBP and LI equations grows faster than the number of independent Feynman integrals one can sooner

\footnotetext{
*Supported by DFG Mercator visiting professorship No. Ha 202/110-1 and Volkswagen Foundation, Contract No. $\mathrm{I} / 77788$.
}

or later obtain an overconstrained system of equations 4.5].

(ii) Using relations that can be obtained by tricks with shifting dimension [6].

(iii) Baikov's method [7.

(iv) Another attempt in this direction is the use of Gröbner basis (see, e.g., [8]).

To evaluate master integrals one uses various methods. In particular, for the evaluation of double and triple boxes the following two methods were successfully applied last years: the method of Mellin-Barnes (MB) representation [9] and differential equations [10. The first of them is based on the following representation

$$
\begin{aligned}
& \frac{1}{(X+Y)^{\lambda}}=\frac{1}{\Gamma(\lambda)} \\
& \quad \times \frac{1}{2 \pi i} \int_{-i \infty}^{+i \infty} \mathrm{d} z \frac{Y^{z}}{X^{\lambda+z}} \Gamma(\lambda+z) \Gamma(-z)
\end{aligned}
$$

which is applied to replace a sum of two terms raised to some power by their products in some powers at the cost of introducing an extra integration. Usually one starts from alpha or Feynman parameters, then introduces, in a minimal way, MB integrations, performs internal integrations over Feynman parameters in terms of gamma functions and obtains a multiple MB representation. It is useful to derive such representation for general powers of the propagators.

The standard procedure of taking residues and shifting contours is used [9, with the goal to obtain a sum of integrals where one may expand integrands in Laurent series in $\epsilon=(4-d) / 2$ (where $d$ is the space-time dimension within dimensional regularization [1]). Then one can use the first 
and the second Barnes lemmas and their corollaries to perform some of the MB integrations explicitly. In the last integrations which usually carry dependence on the external variables, one closes contour in the complex plane and sums up corresponding series. (See [12] for details of the method).

A typical example of application of this method is the evaluation of master integrals for massless on-shell $\left(p_{i}^{2}=0, i=1,2,3,4\right)$ double boxes 915 . where the reduction procedure was performed using shifting dimension 1314, with multiple subsequent applications [16].

\section{Triple boxes and double boxes with one leg off-shell}

The first calculation of massless on-shell triple boxes in dimensional regularization was done in 17, where the Regge asymptotics (in the limit $t / s \rightarrow 0)$ of the master planar triple box shown in Fig. [2] was calculated with the help of the strategy of expansion by regions [18. Later it became possible to evaluate it analytically [19, with the help of a sevenfold MB representation, in terms of harmonic polylogarithms 20. In fact, any mass-

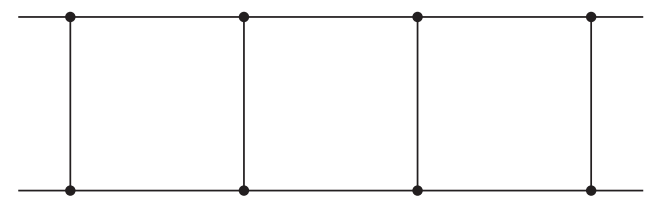

Figure 1. Planar triple box diagram.

less planar on-shell triple box can be evaluated by this procedure so that evaluation of three-loop virtual corrections to various scattering processes is not a miracle.

Let us mention that the necessity to analytically evaluate massless on-shell double and triple boxes arose when studying cross order relations in $N=4$ supersymmetric gauge theories 21. In fact, to check some factorization one needs just one more triple box, in addition to Fig. 1.
The complexity of the problem depends not only on the number of loops but also on the number of parameters. One way to include one more parameter into massless on-shell double boxes is to consider one leg off shell, i.e. $p_{1}^{2}=q^{2} \neq 0$, $p_{i}^{2}=0, i=2,3,4$. This problem was successfully solved last years. The reduction to master integrals was done using Laporta's idea in [5]. Master integrals were calculated using MB representation (first results in 22]) and DE (systematic evaluation in [5]). All results are expressed in terms of two-dimensional harmonic polylogarithms 5 which generalize harmonic polylogarithms. This combination of reduction based on 45] and DE was successfully applied in numerous calculations, e.g., various classes of vertex diagrams [23].

\section{Massive on-shell double boxes}

Another way to include one more parameter into massless on-shell double boxes is to turn to massive on-shell double boxes with one nonzero mass, $p_{i}^{2}=m^{2}, i=1,2,3,4$. Keeping in mind Bhabha scattering let us distinguish two planar and one non-planar diagrams as three basic most complicated types of massive on-shell double boxes. The first planar graph is shown in Fig. 2 For the corresponding general Feynman

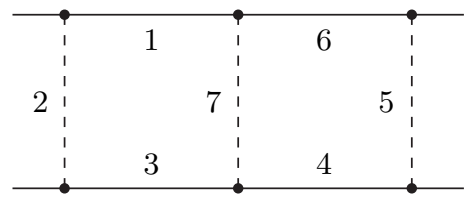

Figure 2. Planar massive on-shell double box of the first type

integral

$$
\begin{aligned}
& B_{P L, 1}\left(a_{1}, \ldots, a_{8} ; s, t, m^{2} ; \epsilon\right) \\
& =\iint \frac{\mathrm{d}^{d} k \mathrm{~d}^{d} l}{\left.\left(k^{2}-m^{2}\right)^{a_{1}}\left[\left(k+p_{1}\right)^{2}\right)\right]^{a_{2}}\left[(k-l)^{2}\right]^{a_{7}}} \\
& \times \frac{1}{\left[\left(k+p_{1}+p_{2}\right)^{2}-m^{2}\right]^{a_{3}}\left[\left(l+p_{1}+p_{2}+p_{3}\right)^{2}\right]^{a_{5}}}
\end{aligned}
$$


$\times \frac{\left[\left(k+p_{1}+p_{2}+p_{3}\right)^{2}\right]^{-a_{8}}}{\left.\left[\left(l+p_{1}+p_{2}\right)^{2}-m^{2}\right)\right]^{a_{4}}\left(l^{2}-m^{2}\right)^{a_{6}}}$,

with an irreducible numerator chosen as $\left(k+p_{1}+\right.$ $\left.p_{2}+p_{3}\right)^{2}$, the following sixfold MB representation was derived in 24:

$$
\begin{aligned}
& B_{P L, 1}\left(a_{1}, \ldots, a_{8} ; s, t, m^{2} ; \epsilon\right) \\
& =\frac{\left(i \pi^{d / 2}\right)^{2}(-1)^{a}}{\prod_{j=2,4,5,6,7} \Gamma\left(a_{j}\right) \Gamma\left(4-a_{4567}-2 \epsilon\right)(-s)^{a-4+2 \epsilon}} \\
& \times \frac{1}{(2 \pi i)^{6}} \int_{-i \infty}^{+i \infty} \mathrm{d} w \prod_{j=1}^{5} \mathrm{~d} z_{j}\left(\frac{m^{2}}{-s}\right)^{z_{15}}\left(\frac{t}{s}\right)^{w} \\
& \times \frac{\Gamma\left(a_{2}+w\right) \Gamma(-w) \Gamma\left(z_{24}\right) \Gamma\left(z_{34}\right)}{\Gamma\left(a_{1}+z_{34}\right) \Gamma\left(a_{3}+z_{24}\right)} \\
& \times \frac{\Gamma\left(4-a_{122388}-2 \epsilon+z_{23}\right)}{\Gamma\left(4-a_{46}-2 a_{57}-2 \epsilon-2 w-z_{1123}\right)} \\
& \times \frac{\Gamma\left(a_{1238}-2+\epsilon+z_{45}\right) \Gamma\left(a_{7}+w-z_{4}\right)}{\Gamma\left(4-a_{1238}-2 \epsilon+w-z_{4}\right)} \\
& \times \frac{\Gamma\left(a_{4567}-2+\epsilon+w+z_{1}-z_{4}\right)}{\Gamma\left(a_{8}-w-z_{234}\right)} \\
& \times \frac{\Gamma\left(a_{8}-z_{234}\right) \Gamma\left(-w-z_{234}-z_{34}\right)}{\Gamma\left(4-a_{13}-2 a_{28}-2 \epsilon+z_{23}-2 z_{5}\right)} \\
& \times \Gamma\left(2-a_{567}-\epsilon-w-z_{12}\right) \\
& \times \Gamma\left(2-a_{457}-\epsilon-w-z_{13}\right) \\
& \times \Gamma\left(2-a_{128}-\epsilon+z_{2}-z_{5}\right) \\
& \times \Gamma\left(4-a_{46}-2 a_{57}-2 \epsilon-2 w-z_{23}\right) \\
& \times \Gamma\left(2-a_{238}-\epsilon+z_{3}-z_{5}\right) \\
& \times \Gamma\left(a_{5}+w+z_{234}\right) \Gamma\left(-z_{1}\right) \Gamma\left(-z_{5}\right)
\end{aligned}
$$

where $a_{4567}=a_{4}+a_{5}+a_{6}+a_{7}, a_{122388}=a_{1}+$ $2 a_{2}+a_{3}+2 a_{8}, z_{1123}=2 z_{1}+z_{2}+z_{3}$, etc.

Analytical evaluation of the master double box $B_{P L, 1}(1, \ldots, 1,0)$ was performed in 24, with a result in terms of polylogarithms. In 25, planar 2-loop box diagrams with one-loop insertion were also by DE. The general MB representation (3) can be used for the evaluation of other master integrals. As an example, a double box with a numerator, $B_{P L, 1}(1, \ldots, 1,-1)$ is evaluated in [26. The finite part of the result in $\epsilon$ includes polylogarithms and HPL

$H\left(-1,0,0,1 ;-\frac{1-1 / \sqrt{1-4 m^{2} / s}}{1+1 / \sqrt{1-4 m^{2} / s}}\right)$.
The second planar graph is shown in Fig. 3 For the corresponding general Feynman integral

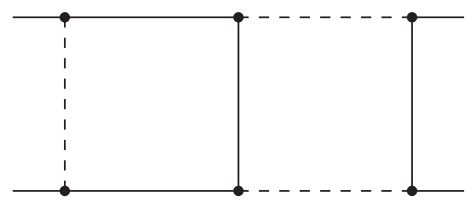

Figure 3. Planar massive on-shell double box of the second type

$$
\begin{aligned}
& B_{P L, 2}\left(a_{1}, \ldots, a_{8} ; s, t, m^{2} ; \epsilon\right) \\
& =\iint \frac{\mathrm{d}^{d} k \mathrm{~d}^{d} l}{\left(k^{2}-m^{2}\right)^{a_{1}}\left(l^{2}\right)^{a_{6}}\left[(k-l)^{2}-m^{2}\right]^{a_{7}}} \\
& \times \frac{1}{\left.\left[\left(k+p_{1}+p_{2}\right)^{2}-m^{2}\right]^{a_{3}}\left[\left(l+p_{1}+p_{2}\right)^{2}\right)\right]^{a_{4}}} \\
& \times \frac{\left[\left(k+p_{1}+p_{2}+p_{3}\right)^{2}\right]^{-a_{8}}}{\left.\left[\left(l+p_{1}+p_{2}+p_{3}\right)^{2}-m^{2}\right]^{a_{5}}\left[\left(k+p_{1}\right)^{2}\right)\right]^{a_{2}}},
\end{aligned}
$$

with the same irreducible numerator, the following sixfold MB representation can be derived:

$$
\begin{aligned}
& B_{P L, 2}\left(a_{1}, \ldots, a_{8} ; s, t, m^{2} ; \epsilon\right) \\
& =\frac{\left(i \pi^{d / 2}\right)^{2}(-1)^{a}}{\prod_{j=2,4,5,6,7} \Gamma\left(a_{j}\right) \Gamma\left(4-a_{4567}-2 \epsilon\right)(-s)^{a-4+2 \epsilon}} \\
& \times \frac{1}{(2 \pi i)^{6}} \int_{-i \infty}^{+i \infty} \prod_{j=1}^{6} \mathrm{~d} z_{j}\left(\frac{m^{2}}{-s}\right)^{z_{5}+z_{6}}\left(\frac{t}{s}\right)^{z_{1}} \\
& \times \prod_{j=1}^{6} \Gamma\left(-z_{j}\right) \frac{\Gamma\left(a_{2}+z_{1}\right) \Gamma\left(a_{4}+z_{24}\right) \Gamma\left(a_{6}+z_{34}\right)}{\Gamma\left(a_{3}-z_{2}\right) \Gamma\left(a_{1}-z_{3}\right)} \\
& \times \frac{\Gamma\left(4-a_{445667}-2 \epsilon-z_{2344}\right)}{\Gamma\left(4-a_{445667}-2 \epsilon-z_{234455}\right)} \\
& \times \frac{\Gamma\left(8-a_{13}-2 a_{245678}-4 \epsilon-z_{11234455}\right)}{\Gamma\left(8-a_{13}-2 a_{245678}-4 \epsilon-z_{1123445566}\right)} \\
& \times \frac{\Gamma\left(2-a_{456}-\epsilon-z_{45}\right) \Gamma\left(2-a_{467}-\epsilon-z_{2345}\right)}{\Gamma\left(a_{45678}-2+\epsilon+z_{2345}\right)} \\
& \times \frac{\Gamma\left(a_{4567}+\epsilon-2+z_{2345}\right)}{\Gamma\left(6-a-3 \epsilon-z_{45}\right)} \\
& \times \Gamma\left(a_{45678}-2+\epsilon+z_{12345}\right)
\end{aligned}
$$


$\times \Gamma\left(4-a_{1245678}-2 \epsilon-z_{12456}\right)$

$\times \Gamma\left(4-a_{2345678}-2 \epsilon-z_{13456}\right)$

$\times \Gamma\left(a-4+2 \epsilon+z_{1456}\right)$.

This multiple MB representation was used in 26 for the evaluation of the master planar double box $B_{P L, 2}\left(1, \ldots, 1,0 ; s, t, m^{2} ; \epsilon\right)$. The result includes polylogarithms, HPL as well as twoparametric integrals of elementary functions. It is not clear at the moment whether these integrals can be written in terms of HPL or $2 \mathrm{dHPL}$ depending on special combinations of $s, t$ and $m^{2}$, or a new class of functions is needed. This result as well as many other results for double and triple boxes mentioned in this short review is confirmed by numerical evaluation by means of a method based on a sector decomposition in the space of alpha parameters [27.

The non-planar graph is shown in Fig. 4

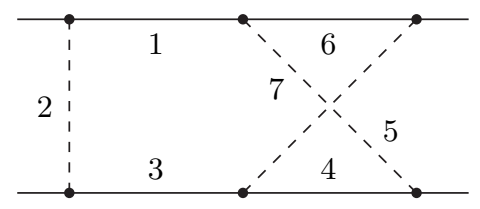

Figure 4. Non-planar massive on-shell double box

The following eightfold MB representation of the general non-planar double box, with the same irreducible numerator as above, can be derived:

$B_{N P}\left(a_{1}, \ldots, a_{8} ; s, t, u, m^{2} ; \epsilon\right)$

$=\frac{\left(i \pi^{d / 2}\right)^{2}(-1)^{a}}{\prod_{j=2,4,5,6,7} \Gamma\left(a_{j}\right) \Gamma\left(4-a_{4567}-2 \epsilon\right)(-s)^{a-4+2 \epsilon}}$

$\times \frac{1}{(2 \pi i)^{8}} \int_{-i \infty}^{+i \infty} \prod_{j=1}^{8} \mathrm{~d} z_{j}\left(\frac{m^{2}}{-s}\right)^{z_{5}+z_{6}}\left(\frac{t}{s}\right)^{z_{7}}\left(\frac{u}{s}\right)^{z_{8}}$

$\times \prod_{j=1}^{7} \Gamma\left(-z_{j}\right) \frac{\Gamma\left(a_{5}+z_{24}\right) \Gamma\left(a_{7}+z_{34}\right)}{\Gamma\left(a_{1}-z_{2}\right) \Gamma\left(a_{3}-z_{3}\right) \Gamma\left(a_{8}-z_{4}\right)}$

$\times \frac{\Gamma\left(2-a_{567}-\epsilon-z_{245}\right) \Gamma\left(2-a_{457}-\epsilon-z_{345}\right)}{\Gamma\left(4-a_{455677}-2 \epsilon-z_{23455}\right)}$

$$
\begin{aligned}
& \times \frac{\Gamma\left(a_{8}+z_{17}-z_{4}\right) \Gamma\left(4-a_{2345678}-2 \epsilon-z_{25678}\right)}{\Gamma\left(6-a-3 \epsilon-z_{5}\right)} \\
& \times \frac{\Gamma\left(a_{28}+z_{178}-z_{4}\right) \Gamma\left(-a_{8}-z_{178}+z_{4}\right)}{\Gamma\left(8-a_{13}-2 a_{245678}-4 \epsilon-z_{2355667788}\right)} \\
& \times \frac{\Gamma\left(4-a_{1245678}-2 \epsilon-z_{35678}\right)}{\Gamma\left(a_{245678}-2+\epsilon+z_{1235788}\right)} \\
& \times \Gamma\left(a_{4567}+\epsilon-2+z_{23458}\right) \Gamma\left(a-4+2 \epsilon+z_{5678}\right) \\
& \times \Gamma\left(a_{245678}-2+\epsilon+z_{12357788}\right) \\
& \times \Gamma\left(4-a_{455677}-2 \epsilon-z_{2344}\right) \\
& \times \Gamma\left(8-a_{13}-2 a_{245678}-4 \epsilon-z_{23445588}\right) .
\end{aligned}
$$

This representation can be used for the evaluation of the non-planar master planar double boxes.

\section{Perspectives}

It has been reported 28] that the reduction of the massive on-shell double boxes relevant to Bhabha scattering can be done using Laporta's algorithm. Master integrals for these three recursion problems were identified. The calculation of these master integrals was performed in all the cases when four indices $a_{i}$ are positive and for some partial cases with five positive indices. In this problem the method of DE meets some complications because differential equations higher that the second order appear for complicated master integrals. Whether or not this obstacle can be overcome is an open question. However, the three general MB representations presented above can be certainly used for the analytical evaluation of the master integrals.

Let us finally characterize advantages of the method based on MB representation:

( $i$ ) The MB representation can be derived for a general Feynman integral corresponding to a given graph, i.e. with general integer powers of the propagators.

(ii) Resolution of singularities in $\epsilon$ is much simpler than in alpha and Feynman parametric integrals.

(iii) After the resolution of singularities in $\epsilon$ one can always switch to numerical evaluation, at least in order to check analytical results. The convergence along imaginary axis is always perfect.

(iv) Automation of calculations based on MB 
representation looks promising.

\section{REFERENCES}

1. G. Passarino and M. Veltman, Nucl. Phys. B160 (1979) 151; S. Actis, A. Ferroglia, G. Passarino, M. Passera and S. Uccirati, hep-ph/0402132,

2. K.G. Chetyrkin and F.V. Tkachov, Nucl. Phys. B192 (1981) 159.

3. T. Gehrmann and E. Remiddi, Nucl. Phys. B580 (2000) 485.

4. S. Laporta, Int. J. Mod. Phys. A15 (2000) 5087.

5. T. Gehrmann and E. Remiddi, Nucl. Phys. B601 (2001) 248, 287.

6. O.V. Tarasov, Nucl. Phys. B480 (1996) 397; Phys. Rev. D54 (1996) 6479.

7. P.A. Baikov, Phys. Lett. B385 (1996) 404; Nucl. Instrum. Methods A389 (1997) 347; V.A. Smirnov and M. Steinhauser, Nucl. Phys. B672 (2003) 199.

8. O.V. Tarasov, hep-ph/0403253 V.P. Gerdt, these proceedings.

9. V.A. Smirnov, Phys. Lett. B460 (1999) 397; J.B. Tausk, Phys. Lett. B469 (1999) 225.

10. A.V. Kotikov, Phys. Lett. B254 (1991) 158; B259 (1991) 314; B267 (1991) 123; E. Remiddi, Nuovo Cim. 110A (1997) 1435; T. Gehrmann and E. Remiddi, Nucl. Phys. B580 (2000) 485.

11. G. 't Hooft and M. Veltman, Nucl. Phys. B44 (1972) 189; C.G. Bollini and J.J. Giambiagi, Nuovo Cim. 12B (1972) 20.

12. V.A. Smirnov, hep-ph/0209177.

13. V.A. Smirnov and O.L. Veretin, Nucl. Phys. B566 (2000) 469.

14. C. Anastasiou, T. Gehrmann, C. Oleari, E. Remiddi and J.B. Tausk, Nucl. Phys. B580 (2000) 577;

15. E.W.N. Glover and M.E. Tejeda-Yeomans, Nucl. Phys. Proc. Suppl. 89 (2000) 196; T. Gehrmann and E. Remiddi, Nucl. Phys. Proc. Suppl. 89 (2000) 251. C. Anastasiou, J.B. Tausk and M.E. Tejeda-Yeomans, Nucl. Phys. Proc. Suppl. 89 (2000) 262.

16. Z. Bern, L. Dixon and A. Ghinculov, Phys. Rev. D63 (2001) 053007; C. Anastasiou,
E.W.N. Glover, C. Oleari and M.E. TejedaYeomans, Nucl. Phys. B601 (2001) 318; B601 (2001) 341; Nucl. Phys. B605 (2001) 486; E.W.N. Glover, C. Oleari and M.E. TejedaYeomans, Nucl. Phys. B605 (2001) 467; Z. Bern, A. De Freitas and L.J. Dixon, JHEP 0109 (2001) 037; Z. Bern, A. De Freitas, L.J. Dixon, A. Ghinculov and H.L. Wong, JHEP 0111 (2001) 031; Z. Bern, A. De Freitas and L.J. Dixon, JHEP 0203 (2002) 018; C. Anastasiou, E.W.N. Glover and M.E. TejedaYeomans, Nucl. Phys. B629 (2002) 255; E.W.N. Glover, hep-ph/0401119

17. V.A. Smirnov, Phys. Lett. B547 (2002) 239.

18. M. Beneke and V.A. Smirnov, Nucl. Phys. B522 (1998) 321; V.A. Smirnov and E.R. Rakhmetov, Teor. Mat. Fiz. 120 (1999) 64; V.A. Smirnov, Phys. Lett. B465 (1999) 226; V.A. Smirnov, Applied Asymptotic Expansions in Momenta and Masses (Springer, Berlin, Heidelberg, 2001).

19. V.A. Smirnov, Phys. Lett. B567 (2003) 193.

20. E. Remiddi and J.A.M. Vermaseren, Int. J. Mod. Phys. A15 (2000) 725.

21. Z. Bern, J.S. Rozowsky, B. Yan, Phys. Lett. B401 (1997) 273; C. Anastasiou, L.J. Dixon, Z. Bern and D.A. Kosower, Phys. Rev. Lett. 91 (2003) 251602; hep-th/0402053

22. V.A. Smirnov, Phys. Lett. B491 (2000) 130; B500 (2001) 330.

23. P. Mastrolia and E. Remiddi, Nucl. Phys. B664 (2003) 341; U. Aglietti and R. Bonciani, Nucl. Phys. B668 (2003) 3; R. Bonciani, P. Mastrolia and E. Remiddi, Nucl. Phys. B661 (2003) 289; B676 (2004) 399; hep-ph/0311145 U. Aglietti and R. Bonciani, hep-ph/0401193 U. Aglietti, R. Bonciani, G. Degrassi and A. Vicini, hep-ph/0404071.

24. V.A. Smirnov, Phys. Lett. B524 (2002) 129.

25. R. Bonciani, A. Ferroglia, P. Mastrolia and E. Remiddi, Nucl. Phys. B681 (2004) 261.

26. G. Heinrich and V.A. Smirnov, to be published.

27. T. Binoth and G. Heinrich, Nucl. Phys. B585 (2000) 741; B680 (2004) 375.

28. J. Gluza, these proceedings. 